\title{
Neuartige Sensorik für die automatisierte Landtechnik \\ Einsatz optischer Sensorik in der Landwirtschaft am Beispiel der Pflugtiefenbestimmung
}

\author{
M. Sc. Simon Becker \\ Karlsruher Institut für Technologie (KIT) - Teilinstitut Mobile \\ Arbeitsmaschinen, Karlsruhe \\ Prof. Dr.-Ing. Marcus Geimer \\ Karlsruher Institut für Technologie (KIT) - Teilinstitut Mobile \\ Arbeitsmaschinen, Karlsruhe
}

\section{Kurzfassung}

Im Rahmen der Digitalisierung der Landwirtschaft sind neue Systeme erforderlich, die bisher manuell erledigte Aufgaben automatisieren können. Zur Erfüllung dieser Anforderung ist die Erfassung der Prozessqualität in landwirtschaftlichen Prozessen notwendig. Hierfür wird Sensorik benötigt, welche bisher in der Landtechnik kaum Verbreitung findet. Dieser Beitrag zeigt am Beispiel der Arbeitstiefenbestimmung eines Pfluges den Einsatz eines Laserscanners und einer Stereokamera am Arbeitsgerät und vergleicht dabei die Ergebnisse dieser beiden Sensoren aus unterschiedlichen Zielgruppen.

\section{Einleitung}

Das Stichwort „Digitalisierung 4.0“ ist aktuell aus allen Richtungen zu hören und beeinflusst auch die Landtechnik. Ein großer Teil der Innovationen kommt dabei nicht mehr aus der klassischen Disziplinen wie des Maschinenbaus, sondern aus der Softwareentwicklung. Hierfür werden entsprechende Plattformen zur Datenverarbeitung und Sensorik benötigt. Im Gegensatz zur Zustandssensorik auf der Maschine hat Prozesssensorik die Aufgabe, Kenngrößen des Arbeitsprozesses zu erfassen. Für die Landtechnik ist Digitalisierung auf der einen Seite nicht neu, auf der anderen Seite gibt es noch offene Themengebiete, die es für die "Landtechnik 4.0“ zu bearbeiten gilt.

Seit der Mechanisierung der Landwirtschaft in der breiten Masse durch die Etablierung von Traktoren hat sich die Aufgabe des Menschen im landwirtschaftlichen Arbeitsprozess gewandelt. War der Mensch anfänglich noch direkter Teil des Prozesses, beispielsweise beim Harken des Bodens oder beim Ziehen eines Pfluges von Hand, haben sich seine Aufgabe 
durch den Einsatz eines Traktors mit Pflug als Anbaugerät hin zu einem Maschinenführer gewandelt. Der Maschinenführer hat die Aufgabe, die Maschine zu steuern und zu überwachen. Gemeinsam mit der Einsatzplanung bildet das Führen der Maschine bis heute die Hauptaufgabe des Landwirtes auf modernen Traktoren.

\section{Digitalisierung in der Landwirtschaft bis heute}

Mit dem Einzug der Elektronik in die Landmaschinen haben auch die ersten digitalen Systeme Einzug gehalten. Angefangen bei der elektronischen Hubwerksregelung EHR [1] bis hin zur automatischen Spurführung mittels GPS-Technik [2] unterstützen die Systeme den Fahrer in immer höherem Maße bei seiner Aufgabe auf der Maschine.

Ein weiterer Baustein der Digitalisierung in der Landtechnik ist der sogenannte Isobus [3]. Diese Kommunikationsschnittstelle ermöglicht den Datenaustausch zwischen Traktor und Arbeitsgerät sowie die bidirektionale Steuerung beider Maschinen. Hiermit wurde bereits der erste Grundstein für die Vernetzung von Maschinen gelegt. Diese Schnittstelle wurde verwendet, um beim Kreiseleggen den Traktor abhängig des durch eine Stereokamera erfassten Arbeitsergebnisses zu steuern [4].

\section{Automatisierung der Landwirtschaft}

Um die Automatisierung in der Landtechnik weiter voranzutreiben, ist es notwendig zeitgleich die Maschine, den Fahrer und den Arbeitsprozess als Gesamtprozess zu betrachten. Abbildung 1 stellt den Informationsfluss zwischen dem landwirtschaftlichen Arbeitsprozess, dem Traktor und dem Fahrer dar. Der Fahrer beobachtet den Arbeitsprozess mit seinen Sinnen (Sehen, Hören, Fühlen), verarbeitet diese Informationen und leitet daraus Handlungen ab, die er mit seinen Gliedmaßen ausführt um die Maschine zu steuern [5].

Die Hauptaufgaben lassen sich folgendermaßen zusammenfassen:

- Überwachen des Arbeitsprozesses

- Entscheidungen ableiten

- Ansteuern der Maschine 


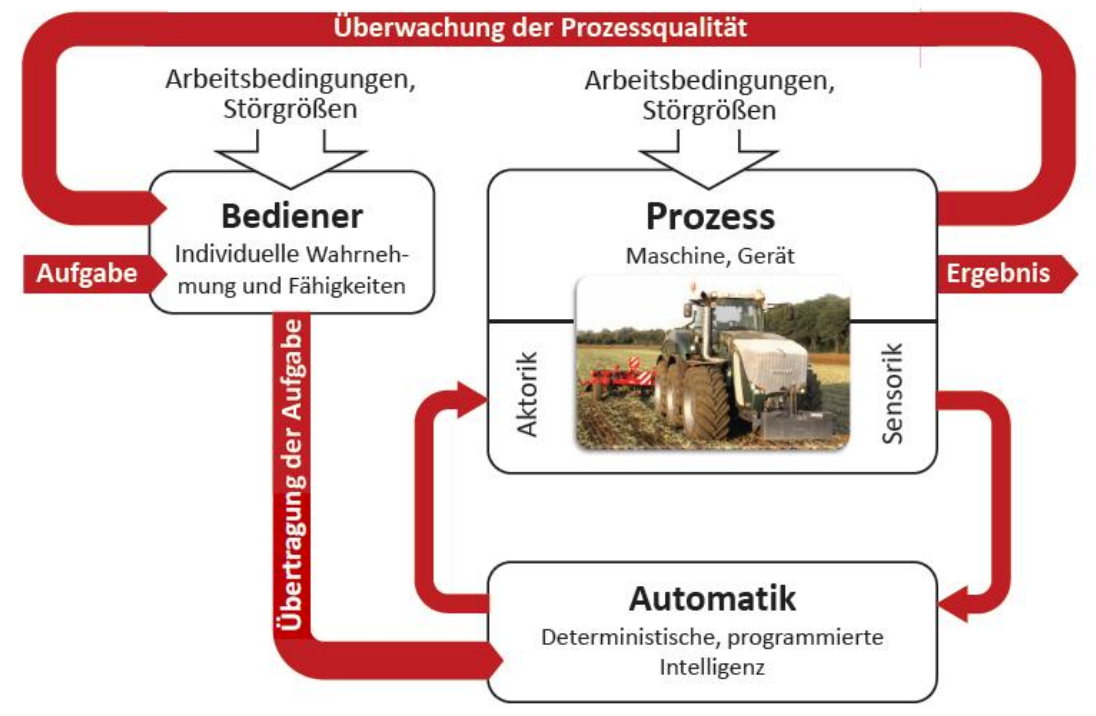

Bild 1: Der Bediener im Arbeitsprozess [6]

Die zuletzt genannte Aufgabe des Steuerns ist bei in der Regel elektronisch gesteuerten Maschinen durch Zugriff auf den CAN-Bus relativ einfach lösen. Um Entscheidungen für die Ansteuerung der Maschine zu generieren sind Systeme erforderlich, die wie der Fahrer unterschiedliche Eingangsparameter aufnehmen, verarbeiten und daraus Steuerungsparameter ableiten. Viele wichtige Eingangsparameter werden bereits elektronisch erfasst, beispielsweise Fahrgeschwindigkeit, Schlupf, Zugkraft oder Position des Heckkrafthebers. Andere Prozessinformationen werden jedoch von dem Fahrer manuell erfasst. Durch Beobachtung des Arbeitsprozesses ermittelt er mithilfe seiner Prozesskenntnis die Arbeitsqualität. Ziel dieses Beitrags ist es, anhand der Arbeitstiefenbestimmung beim Pflügen zu zeigen, wie mit Hilfe von moderner Sensorik die elektronische Erfassung dieser Parameter aussehen könnte. Einige allgemeine Beispiele für die daraus zu gewinnenden Parameter beim Pflügen wären:

- Arbeitstiefe

- Parallelstellung des Pflugs zum Boden

- Gutfluss an den Pflugscharen

- Krümeligkeit des gewendeten Bodens

- Anteil organischer Bestandteile im gewendeten Boden

- Grad der Furchenräumung

In dem folgenden Abschnitt wird nun die Arbeitstiefenbestimmung beispielhaft gezeigt werden. 


\section{Arbeitstiefenbestimmung}

\section{Versuchsaufbau}

Folgender Versuchsaufbau wurde zur Arbeitstiefenbestimmung gewählt:

- Fendt Vario 412

- Kverneland 4-Schar Drehpflug

- Intel Realsense D435 Stereokamera

- $\quad$ Sick LMS 511 Lite Laserscanner

Die genannten Sensoren wurden mittels Aluprofilschienen auf dem Pflugrahmen so befestigt, dass sie, wie in Abbildung 2 zu sehen, senkrecht nach unten zeigend die Pflugfurche direkt nach dem letzten Schar aufnehmen können.
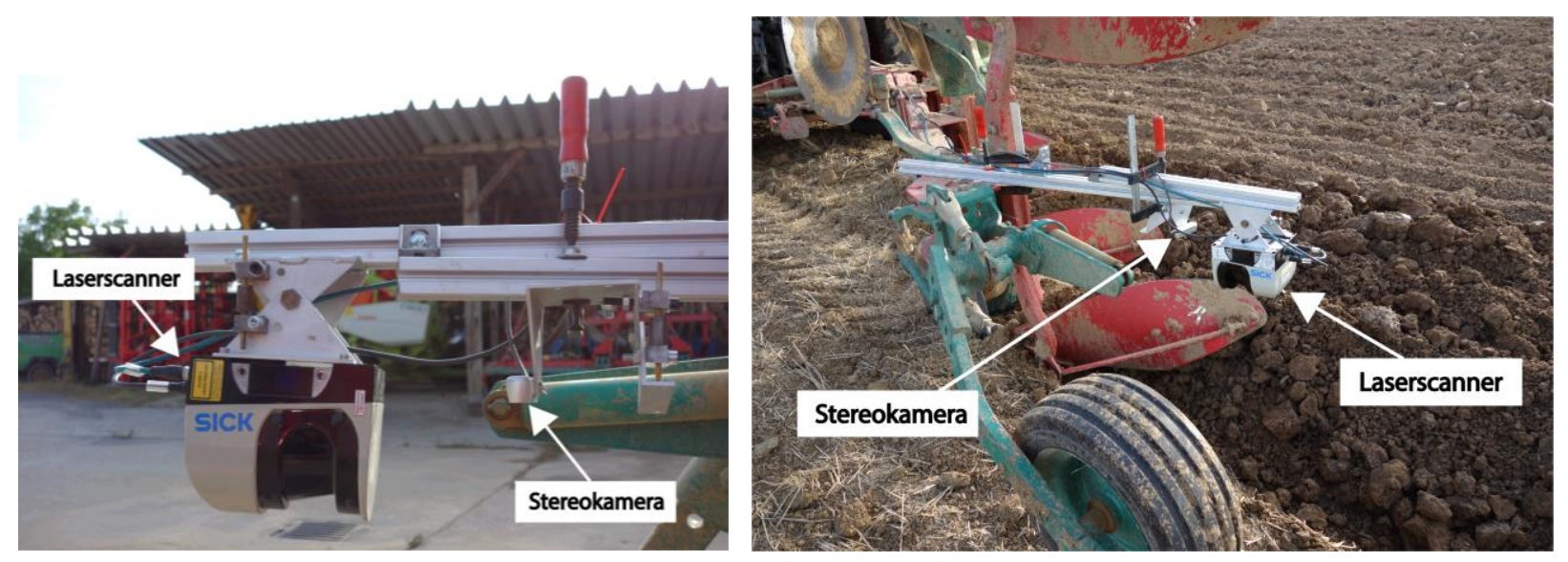

Bild 2 a) und b): Laserscanner und Stereokamera auf Aluprofilschienen am Pflug befestigt

\section{Arbeitstiefenbestimmung mit Laserscanner}

In Bild 3 a) ist der nicht aufbereitete Laserscan einer Pflugfurche zu sehen. Durch die eingestellte Scanrichtung senkrecht nach unten handelt es sich um den Querschnitt der Pflugfurche. Die senkrechte Kontur im linken Bildbereich bis zu einer Breite von $-0,5 \mathrm{~m}$ stellt das Stützrad des Pfluges dar. Daneben befindet sich bis zu einer Breite von $-0,25 \mathrm{~m}$ der unbearbeitete Bodenbereich. Direkt daran schließt sich die Pflugfurche bis $0,5 \mathrm{~m}$ Breite an, 


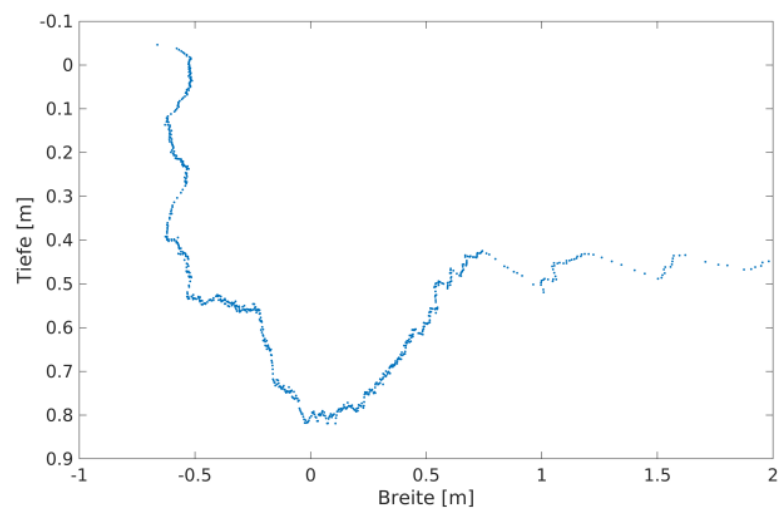

Bild 3 a): Laserscan einer Pflugfurche

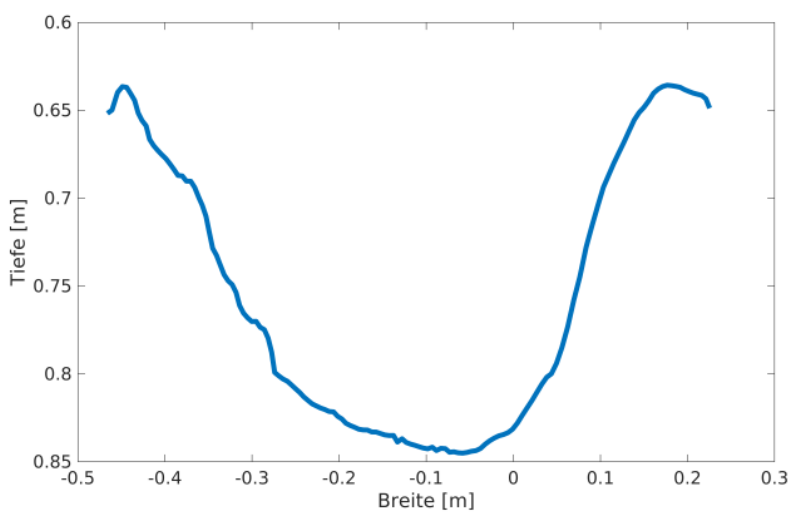

b) Stereokamerascan einer Pflugfurche

außerdem ist rechts daneben der durch drei der Schare umgewendete Boden zu erkennen. Zur Berechnung der Arbeitstiefe wurde zunächst, wie in Bild 4 zu sehen, aus den Messdaten eine charakteristische Referenzkurve ausgewählt. Dabei ist nicht deren absolute Tiefe, sondern die Gestalt entscheidend.

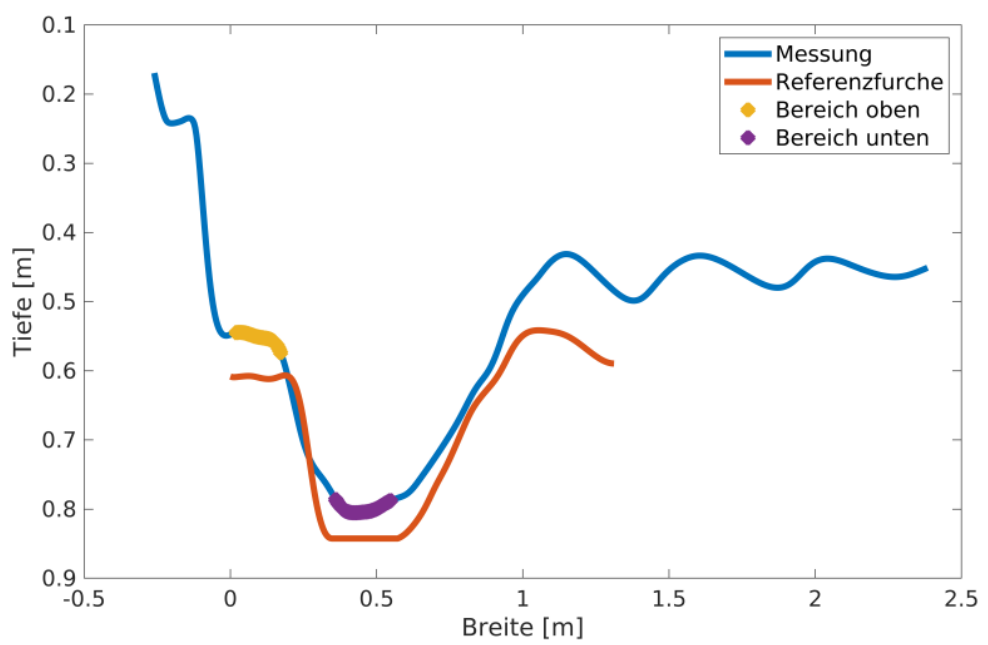

Bild 4: Mit Referenzfurche überlagerter Laserscan sowie oberer und unterer Bereich zur Bestimmung der Arbeitstiefe

In einem ersten Schritt wird der Laserscan solange seitlich verschoben, bis er mit der Referenzkurve möglichst deckungsgleich ist. Somit ist die Position der Furche im verwendeten Koordinatensystem immer gleich und unabhängig von Verschiebungen des Laserscanners relativ zum Pflug. Deshalb können Punkte basierend auf der Breitenkoordinate den Bereichen „oben“ und „unten“ zugeordnet werden. Im zweiten Schritt werden Punkte aus den in Abbildung 4 gekennzeichneten Intervallen je einem oberen und unteren Bereich zugeordnet. 
Abschließend wird daraus je ein Mittelwert gebildet. Der Abstand zwischen oberem und unterem Mittelwert ergibt somit die Pflugtiefe.

Während Laserscanner mit einigen tausend Euro Einkaufspreis dem professionellen Bereich entstammen, wurden die gleichen Versuche parallel mit einer Stereokamera aus dem Consumerbereich gefahren. Hierfür wurde eine Intel Realsense Stereokamera mit einem Einkaufspreis von ca. $300 €$ gewählt. Abbildung 5 zeigt das Ergebnis der Aufnahme.

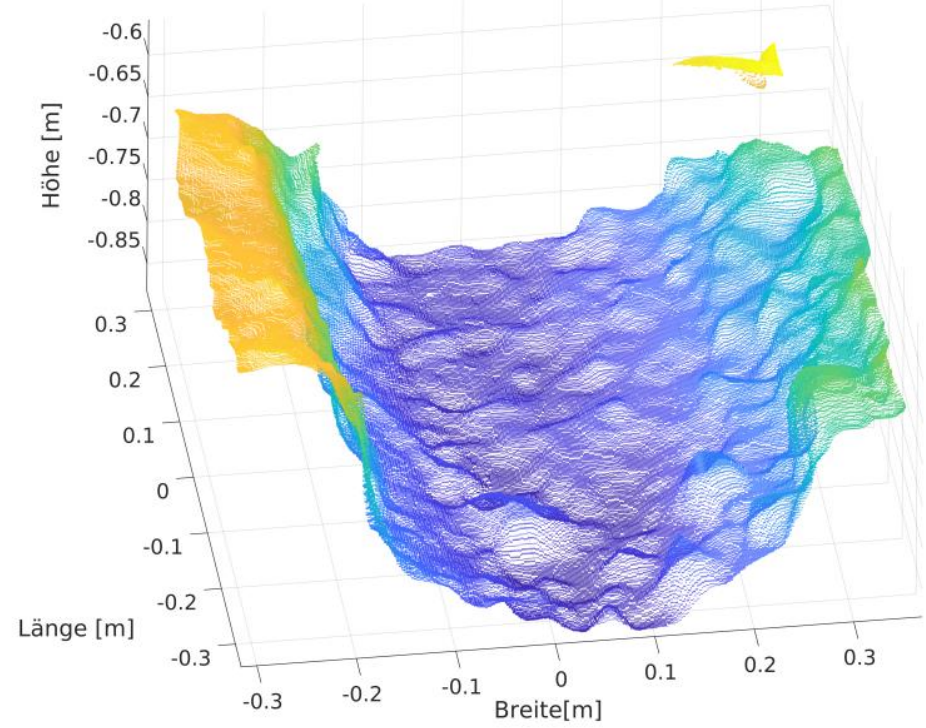

Bild 5: Stereokamerabild der Pflugfurche

Das Bild 3 b) zeigt den Querschnitt der Pflugfurche, welcher aus dem Tiefenbild der Stereokamera berechnet wurde. Hierzu wurden die Tiefenwerte entlang der Pflugfurche über die Aufnahmelänger der Stereokamera gemittelt.

Auch für die Stereokamera wird das gleiche Verfahren zur Arbeitstiefenbestimmung durchgeführt. Abbildung 6 zeigt einen Vergleich der Ergebnisse der Tiefenbestimmung von Laserscanner und Stereokamera über einen Weg von ca. 110 Metern. Zu sehen ist, dass zwischen den beiden Sensoren eine Abweichung von ca. $4 \mathrm{~cm}$ festzustellen ist. Dies lässt sich auf eine noch nicht erfolgte Kalibrierung zwischen den Sensoren zurückführen. Gleichzeitig lässt sich feststellen, dass das Ergebnis des Laserscanners ein höheres Rauschen aufweist. Dies liegt in dem schmaleren Messbereich verglichen zur Stereokamera begründet, wodurch einzelne Erdklumpen einen höheren Einfluss auf die Messungen haben. 


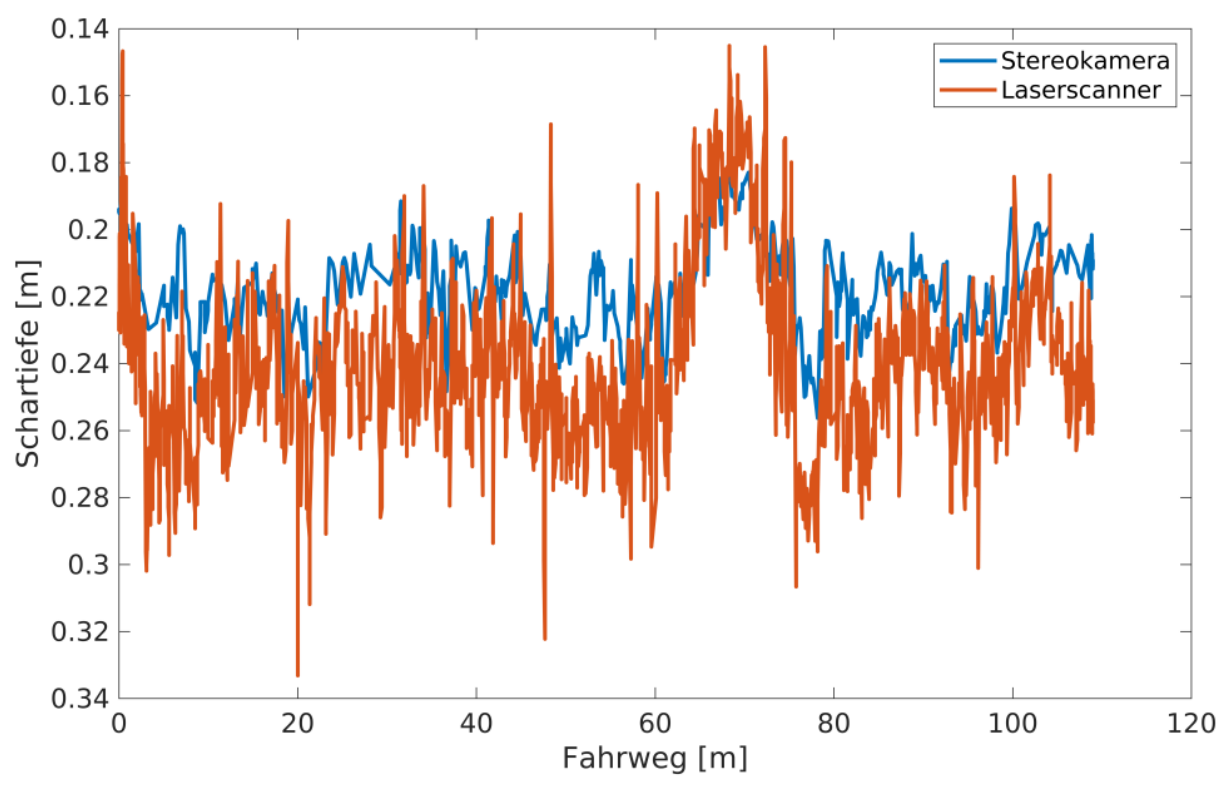

Bild 6: Ergebnis der Arbeitstiefenbestimmung über den Weg

\section{Zusammenfassung und Ausblick}

In diesem Betrag wurde die Bedeutung von Prozesssensorik für die automatisierte Landwirtschaft der Zukunft dargestellt. Als Beispiel wurde die Arbeitstiefenbestimmung eines Pfluges mittels Laserscanner und Stereokamera gezeigt. Diese haben gezeigt, dass mit einer Stereokamera aus dem Consumerbereich sehr gute Ergebnisse erzielt werden können.

Für die Zukunft sind weitere Anwendungsszenarien denkbar. Durch die Kombination der Arbeitstiefenbestimmung mit beispielsweise Neigungssensoren ist eine Bestimmung der Pflugausrichtung möglich und damit implizit auch die Arbeitstiefe der vorderen Schare. Diese Informationen können in Zukunft als Eingangsparameter für eine automatisierte Prozesssteuerung dienen. 


\section{Literaturverzeichnis}

[1] Weisbrodt, J. u. Köller, K.: Vergleich von doppelt und einfach wirkender EHR in der Bodenbearbeitung. 364-367 Seiten / LANDTECHNIK, Bd. 65, Nr. 5 (2010) (2010)

[2] Landerl, G.: Untersuchungen zum Nutzen und zu Genauigkeiten von GPS-gestützten Parallelfahrsystemen (Lenkhilfe, Lenkassistent und Lenkautomat) bei Traktoren, Universität für Bodenkultur Diplomarbeit. Wien

[3] Steffen Hermann: Dem Isobus-Standard gehört die Zukunft. Branche zieht im elektroniksektor an einem Strang - Weltweite Akzeptanz nimmt zu. agrarzeitung 2011 (2011) 10

[4] Peter Riegler-Nurscher, Jürgen Karner, Josef Huber, Gerhard Moitzi, Helmut Wagentristl, Markus Hofinger, Heinrich Prankl: A system for online control of a rotary harrow using soil roughness detection based on stereo vision

[5] Einflüsse der Betriebseffizienz auf die $\mathrm{CO}_{2} \mathrm{e}$-Emissionen und die entstehenden Änderungen mit automatisierten Arbeitsmaschinen, Ays, I., Becker, S. u. Geimer, M.

[6] Pichlmaier, B.: Traktionsmanagement für Traktoren. 2012 